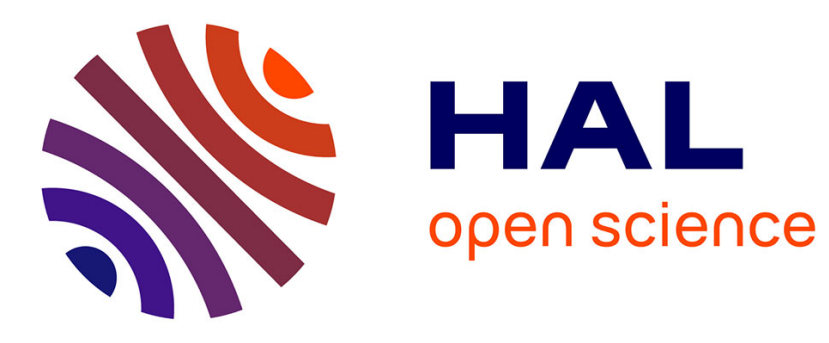

\title{
RF Linewidth in Monolithic Passively Mode-Locked Semiconductor Laser
}

\author{
Fabien Kéfélian, Shane O’Donoghue, Maria Teresa Todaro, John G. \\ Mcinerney, Guillaume Huyet
}

\section{- To cite this version:}

Fabien Kéfélian, Shane O’Donoghue, Maria Teresa Todaro, John G. Mcinerney, Guillaume Huyet. RF Linewidth in Monolithic Passively Mode-Locked Semiconductor Laser. IEEE Photonics Technology Letters, 2008, 20 (16), pp.1405-1407. 10.1109/LPT.2008.926834 . hal-01075175

\section{HAL Id: hal-01075175 \\ https://hal.science/hal-01075175}

Submitted on 16 Oct 2014

HAL is a multi-disciplinary open access archive for the deposit and dissemination of scientific research documents, whether they are published or not. The documents may come from teaching and research institutions in France or abroad, or from public or private research centers.
L'archive ouverte pluridisciplinaire HAL, est destinée au dépôt et à la diffusion de documents scientifiques de niveau recherche, publiés ou non, émanant des établissements d'enseignement et de recherche français ou étrangers, des laboratoires publics ou privés. 


\title{
RF Linewidth in Monolithic Passively Mode-Locked Semiconductor Laser
}

\author{
Fabien Kéfélian, Shane O’Donoghue, Maria Teresa Todaro, John G. McInerney, and Guillaume Huyet
}

\begin{abstract}
We have analyzed theoretically and experimentally the linewidth of the first harmonic of the photocurrent (radio-frequency ( $R F$ ) linewidth) in monolithic passively mode-locked semiconductor lasers. Due to the absence of restoring force, the timing jitter is directly related to the RF linewidth, avoiding possible underestimations made with conventional methods of phase noise measurement. The RF linewidth is also analytically related to the pulse characteristics using Haus's model. The timing stability performance of a promising two-section quantum-dot laser is presented using RF linewidth measurements. Experimental evolution of the RF linewidth with power and pulsewidth is finally compared to the analytical expression.
\end{abstract}

Index Terms-Mode-locked laser, quantum dot, semiconductor laser, timing jitter.

\section{INTRODUCTION}

$\mathbf{H}$ IGH repetition rate optical pulse sources are needed for diverse applications including optical sampling, clocking, optical time-division multiplexing, clock recovery and frequency conversion, radar and remote sensing, and medical diagnostics. In all these applications, few picoseconds pulsewidth and subpicosecond timing jitter is essential. Monolithic mode-locked semiconductor lasers are increasingly attractive to provide stable and reliable pulse trains with multigigahertz repetition rates [1]. Passive mode-locking can be achieved simply in these lasers by creating two electrically isolated sections and applying a reverse voltage to one of them to obtain an intracavity saturable absorber. Passively mode-locked lasers (PMLLs) yield much shorter pulses and higher repetition rate than actively mode-locked lasers, without any external electrical oscillator.

Understanding, improving, and controlling the timing jitter is essential to improve performance, manufacturability, and widespread use of mode-locked lasers in real applications. In experimental characterization of PMLL, timing jitter is generally

Manuscript received February 25, 2008; revised May 16, 2008. This work was supported by the Science Foundation Ireland Grant sfi/01/fi/co.

F. Kéfélian was with Tyndall National Institute, Cork, Ireland and Cork Institute of Technology, Cork, Ireland. He is now with the Laboratoire de Physique des Lasers, Université Paris-XIII, Villetaneuse 93430, France.

S. O'Donoghue is with Tyndall National Institute, Cork, Ireland and Cork Institute of Technology, Cork, Ireland. He is also with the Departement of Physics, University College Cork, Cork, Ireland.

M. T. Todaro was with Tyndall National Institute, Cork, Ireland and the Department of Physics, University College Cork, Cork, Ireland. She is now with the National Nanotechnology Laboratory, Consiglio Nazionale delle Ricerche/Istituto Nazionale per la Fisica della Materia and Universita del Salento, 73100 Lecce, Italy.

J. G. McInerney is with Tyndall National Institute, Cork, Ireland and the Department of Physics, University College Cork, Cork, Ireland.

G. Huyet is with Tyndall National Institute, Cork, Ireland and Cork Institute of Technology, Cork, Ireland.

Digital Object Identifier 10.1109/LPT.2008.926834 given in terms of integrated phase noise power spectral density (PSD) measured from the photocurrent, like in von der Linde's method [2]. However, although this method is well suited for actively mode-locked lasers, it should be applied only with caution to PMLL.

Due to the intrinsic phase noise properties of semiconductor PMLL [3], the study of the linewidth of the first harmonic of the photocurrent (radio-frequency (RF) linewidth) can provide a simpler and more appropriate way to characterize the timing stability of these pulse sources. Here we first study the relation between RF linewidth measurement and timing jitter, and give an analytical expression for the RF linewidth. Then we present the timing stability performance of a promising high repetition rate quantum-dot PMLL using measurements of the RF linewidth and compare its evolution with power and pulsewidth to the analytical expression.

\section{RF LiNEWIDTH AND TIMING JiTtER RELATIONS}

Timing jitter is expressed by two quantities, the integrated root-mean-square (rms) timing jitter [2]

$$
\sigma_{T}^{i}\left(f_{u}, f_{d}\right)=\frac{T_{R}}{2 \pi} \sqrt{\int_{f_{d}}^{f_{u}} S_{\phi_{\mathrm{RF}}}(f) d f}
$$

and the pulse-to-pulse rms timing jitter [4]

$$
\sigma_{T}^{p p}(N)=\frac{T_{R}}{\pi} \sqrt{\int_{0}^{+\infty} \sin ^{2}\left(\pi f N T_{R}\right) S_{\phi_{\mathrm{RF}}}(f) d f}
$$

where $f_{u}$ and $f_{d}$ are the upper and lower frequencies of integration, respectively, $T_{R}$ the period of the pulse train, $N$ the number of periods between the two compared pulses and $S_{\phi_{\mathrm{RF}}}(f)$ the single sideband (SSB) PSD of the RF phase noise.

The integrated rms timing jitter is generally measured from the photocurrent PSD using von der Linde's method [2]. However, it is important to note that actively and passively modelocked lasers exhibit very different photocurrent PSD [3], [5]. In the active case, the spectral shape at each harmonic is composed of a narrow spike on a pedestal. Due to locking to the stable external oscillator, the timing fluctuations of the pulse train are small. Consequently, using a first-order approximation, the PSD of the RF phase noise can be obtained from the PSD of the photocurrent. This is not the case with passive mode-locking, as a relatively broad lineshape, instead of the narrow spike, exists at each harmonic. Driven by the spontaneous emission and technical noise (e.g., pump or control flucations), and without external stabilization, the pulse train has large timing fluctuations and so the RF phase exhibits statistical properties similar to those of the optical phase, such as nonstationarity. Consequently, the first-order approximation used in [2] is not automatically valid. 
The pulse-to-pulse rms timing jitter has an advantage in that it can be measured directly by optical cross-correlation using second-harmonic generation in a nonlinear crystal. This, however, requires very specific materials, mountings, optical alignment, precise temperature control, and mechanical scanning. Moreover, the measurement error becomes very large when the timing jitter is much smaller than the autocorrelation width [4].

Previous works [3], [5] have shown that in PMLL the RF frequency noise is induced by the relatively broadband spontaneous emission and is consequently essentially white. This leads to a Lorentzian-shaped PSD of the first harmonic of the photocurrent. Consequently, the full-width at half-maximum of the Lorentzian $\Delta \nu_{\mathrm{RF}}$ determines completely the SSB PSD of the RF phase noise [6]

$$
S_{\phi_{\mathrm{RF}}}(f)=\frac{\Delta \nu_{\mathrm{RF}}}{\pi f^{2}}
$$

the pulse to pulse rms timing jitter [using (1) and (3)]

$$
\sigma_{T}^{p p}(N)=T_{R} \sqrt{\frac{\Delta \nu_{\mathrm{RF}} N T_{R}}{2 \pi}}
$$

and the integrated rms timing jitter [using (2) and (3)]

$$
\sigma_{T}^{i}\left(f_{u}, f_{d}\right)=\frac{T_{R} \sqrt{\Delta \nu_{\mathrm{RF}}}}{2 \pi^{3 / 2}} \sqrt{\frac{1}{f_{d}}-\frac{1}{f_{u}}} .
$$

In this case, von der Linde's method corresponds to the approximation of the Lorentzian by $1 / f^{2}$, which is only valid for $f \gg \Delta \nu_{\mathrm{RF}} / 2$ and can consequently lead to large underestimation of the integrated rms timing jitter when $f_{d}$ is small.

Instead of calculating the RF phase noise PSD from the photocurrent, here we propose that it is advantageous to measure the linewidth of the Lorentzian and thus to determine the rms timing jitter using (4) or (5). To fit accurately the photocurrent PSD with a Lorentzian, it is necessary to perform two measurements. First, a measurement of the total RF power in the Lorentzian, using a large frequency span and a large resolution bandwidth (typically $100-\mathrm{MHz}$ span and 3-MHz bandwidth), and second, a high resolution measurement of the PSD by scanning a short span and narrow resolution bandwidth (typically $5-\mathrm{MHz}$ span and 3-kHz bandwidth). In such a way, only two parameters have to be extracted from the fitting: the central frequency and the 3 -dB RF linewidth. This procedure reduces greatly the measurement error of the RF linewidth. This method can be viewed as a modified version of von der Linde's method in two ways: first the power in the RF carrier, which does not exist in PMLL, is replaced by the total RF spectral power, and second the RF phase noise PSD is not approximated by the photocurrent PSD but is analytically derived from fitting the Lorentzian.

To validate this method, it is essential to check that the pulse train timing jitter is dominated by the spontaneous emission noise, i.e., that the Lorentzian fitting is accurate on a relatively large offset frequency span $\left[0-f_{0}\right]$. The $1 / f^{2}$ contribution will represent more than $90 \%$ of the rms integrated timing jitter if $f_{0}>10 \sqrt{f_{u} f_{d}}$, which gives a span of $100 \mathrm{MHz}$ for an integration band [100 kHz-1 GHz]. The scope for this checking will be usually limited by the shot-noise level, but by using higher harmonics, permitted by the measurement bandwidth (e.g., of the spectrum analyzer), the fidelity can be improved by $6 \mathrm{~dB}$ per harmonic.
This measurement of timing jitter using the photocurrent spectrum can be perturbed by amplitude noise. As with active mode-locking [2], a distinction between phase noise and amplitude noise is made by using higher harmonics of the photocurrent. Also, in PMLL, the amplitude noise is dominated by the phase noise in a large offset frequency bandwidth [7] and consequently has no influence on the RF linewidth.

\section{RF LINEWIDTH AND PULSE CHARACTERISTICS}

The following expression for the RF linewidth of PMLL is derived using the expression of the timing jitter PSD calculated form Haus's equation in [4] (with the corrections proposed in [8]) and the property that the linewidth is formally given by the frequency noise PSD at $0 \mathrm{~Hz}$ [6]

$$
\Delta \nu_{\mathrm{RF}}=2 \frac{\tau^{2}}{T_{R}^{2}}\left(0.53+0.01 \frac{D^{2} \Delta f_{g}^{4}}{g^{2}}\right) \Delta \nu_{\mathrm{ST}}+\frac{\gamma^{2}}{T_{R}^{2}} \mathrm{RIN}
$$

where $\tau$ is the full-width at half-maximum of the hyperbolic secant pulse, $g$ the saturated intensity gain per round-trip, $D$ the net group delay dispersion in $s^{2}, \Delta f_{g}$ the gain bandwidth, $\Delta \nu_{\mathrm{ST}}$ the Schawlow-Townes optical linewidth, $\gamma$ the coupling factor from the energy to the timing fluctuations introduced in [4], and RIN the relative intensity noise at low frequency. Due to the high level of spontaneous emission in diode lasers, technical noise is not considered here.

The RF linewidth is the sum of three contributions, the direct projection of the amplified spontaneous emission, the conversion of the optical frequency fluctuations in RF frequency noise by the dispersion, and the conversion of the RIN in RF frequency noise due to fast gain changes. For typical parameter values (as in [4, Table I]), the second contribution is negligible. The optical linewidth enhancement factor has no direct influence on the RF linewidth. Indeed, assuming the dispersion to be slowly varying over the small optical spectral ranges considered, the linewidth enhancement factor should be frequency-independent and therefore the carrier-induced optical phase noise is identical for all optical modes and consequently has no influence on the phase difference and on the RF phase.

\section{EXPERIMENTS AND DISCUSSION}

We have applied the relation between timing jitter and RF linewidth to characterize a two-section quantum-dot PMLL. Quantum-dot lasers have demonstrated numerous advantages over bulk and quantum-well lasers such as low wavelength chirp, low threshold currents, and reduced linewidth enhancement factor. In particular, broad spectra due to dot size dispersion and easy absorption saturation make them well suited to mode-locking and broadband applications.

The devices studied have room temperature emission at $1.3 \mu \mathrm{m}$ and are similar to the laser described in [9]. Three lasers, (MT1, MT5, MT6), were cleaved with different lengths giving repetition rates of, respectively, $19.2,16.7$, and $16.1 \mathrm{GHz}$ with absorber section fractional length of $10 \%, 10 \%$, and $30 \%$, respectively. In (4) and (5), we have shown that integrated and pulse-to-pulse rms timing jitter are proportional to the square root of the RF linewidth. To study the timing stability of these lasers, we have thus measured the RF linewidth as a function of the gain current for different absorber voltages. For the range of currents considered, the PSD of the photocurrent 


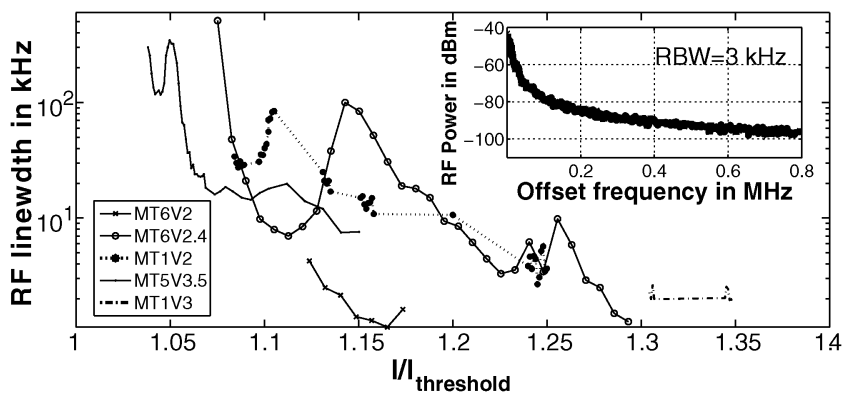

Fig. 1. RF linewidth as a function of the normalized gain current for different lasers and absorber voltages. Inside, right side of the RF spectrum of the photocurrent.

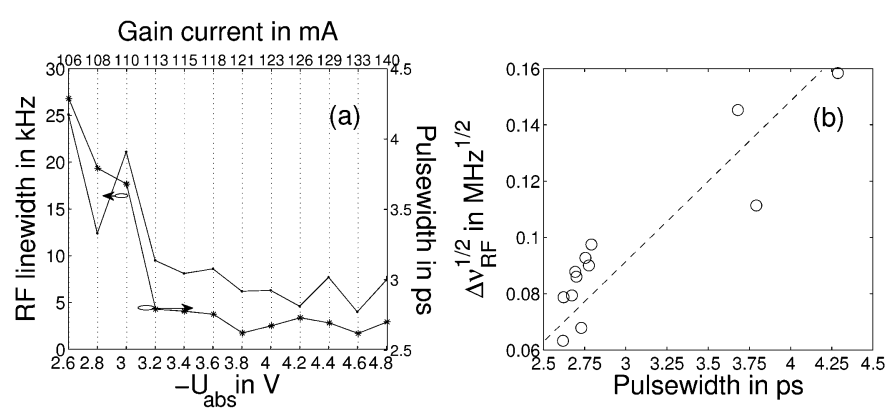

Fig. 2. RF linewidth measurement at constant power.

exhibits a clear Lorentzian shape over more than $50 \mathrm{~dB}$ (see Fig. 1). Moreover, previous measurements on a similar device with an $8 \mathrm{GHz}$ of repetition rate, using the fourth harmonic photocurrent, also demonstrated excellent $1 / f^{2}$ slope between 1 and $100 \mathrm{MHz}$ [9]. Since the threshold current is different for the different laser lengths and the different absorber voltages, the results are presented in Fig. 1 as a function of the gain current normalized by the threshold current. The results show that the RF linewidth is, for a large range of currents, below $10 \mathrm{kHz}$ and reaches $1.6 \mathrm{kHz}$ with MT6 at $140 \mathrm{~mA}$ and $-2 \mathrm{~V}$ with a corresponding pulsewidth of 2 ps and a peak power of $70 \mathrm{~mW}$ per facet. This RF linewidth corresponds, according to (4) and (5), to a pulse-to-pulse timing jitter of $8 \mathrm{fs} /$ cycle, an integrated timing jitter in the range $[20 \mathrm{kHz}-80 \mathrm{MHz}]$ of $1.6 \mathrm{ps}$ and in $[100 \mathrm{kHz}-1 \mathrm{GHz}]$ of $715 \mathrm{fs}$.

We also compared the experimental evolution of the RF linewidth as a function of power and pulsewidth to the behaviour expected from (6). The optical power emitted by the laser increases linearly with the gain current. Consequently, the optical linewidth, according to the Schawlow-Townes equation, and the RIN, should decrease by an amount inversely proportional to the gain current change and, therefore, the RF linewidth should also follow the same behaviour $\left(\Delta \nu_{\mathrm{RF}} \propto 1 / P_{\mathrm{opt}}\right)$. This is roughly observed for currents between 1.15 and 1.3 times the threshold and also very close to the threshold current. However, for each case studied, the RF linewidth also rebroadens one or several times between 1.05 and 1.15 times threshold. From (6), a $\Delta \nu_{\mathrm{RF}} \propto \tau^{2}$ scaling is expected. We have consequently performed measurements of the RF linewidth for different pulsewidths, keeping the average optical power constant by changing simultaneously the gain current and the voltage applied to the absorber section of the laser MT5. Fig. 2(a) presents the values of the RF linewidth and the pulsewidth for different currents, in the range 1.15-1.25 times the threshold, and the corresponding voltage. The square root of the RF linewidth is plotted against the pulsewidth in Fig. 2(b). The results confirm only approximately the expected behavior.

The measured variation of the RF linewidth with power and pulsewidth provide approximate confirmation of our expression (6) based on the first-order expansion of Haus's equation with Langevin forces. We believe that excitation of high-order modes or supermodes [10], which are not included in Haus's model, could have a significant contribution to the timing jitter. Finally, it is intended to compare experimental results with the theoretical expression, which will require further data.

\section{CONCLUSION}

In summary, we have demonstrated a new approach to characterize the timing stability of PMLL using the RF spectrum. Application of the method to InGaAs quantum-dot lasers at $1300 \mathrm{~nm}$ demonstrates the existence of states with very narrow $(<2 \mathrm{kHz}) \mathrm{RF}$ linewidths and these will be the subjects of further study. Additional data are also required to identify any additional contributions to the pulse timing jitter.

\section{REFERENCES}

[1] K. A. Williams, M. G. Thompson, and I. H. White, "Long-wavelength monolithic mode-locked diode lasers," New J. Physic, vol. 6, no. 1, p. 179, 2004.

[2] D. von der Linde, "Characterization of the noise in continuously operating mode-locked lasers," Appl. Phys. B, vol. 39, no. 4, pp. 201-217, Apr. 1986.

[3] H. A. Haus and A. Mecozzi, "Noise of mode-locked lasers," IEEE J. Quantum Electron., vol. 29, no. 3, pp. 983-996, Mar. 1993.

[4] L. A. Jiang, M. E. Grein, H. A. Haus, and E. P. Ippen, "Noise of modelocked semiconductor lasers," IEEE J. Sel. Topics Quantum Electron. vol. 7, no. 2, pp. 159-167, Mar. 2001.

[5] D. Eliyahu, R. A. Salvatore, and A. Yariv, "Effect of noise on the power spectrum of passively mode-locked lasers," J. Opt. Soc. Amer. B, Opt. Phys., vol. 14, no. 1, pp. 167-174, Jan. 1997.

[6] Y. Yamamoto, "AM and FM quantum noise in semiconductor lasers," IEEE J. Quantum Electron., vol. QE-19, no. 1, pp. 34-46, Jan. 1982.

[7] J. Mulet, J. Moerk, and M. Kroh, "Identification of amplitude and timing jitter in external-cavity mode-locked semiconductor lasers," in CLEO/IQEC, San Francisco, CA, May 16-21, 2004.

[8] R. Paschotta, "Noise of mode-locked lasers," Appl. Phys. B, vol. 79, pp. 153-173, 2004.

[9] M. T. Todaro, J.-P. Tourrenc, S. P. Hegarty, C. Kelleher, B. Corbett, G. Huyet, and J. G. McInerney, "Simultaneous achievement of narrow pulsewidth and low pulse-to-pulse timing jitter in $1.3 \mu \mathrm{m}$ passively mode-locked quantum-dot lasers," Opt. Lett., vol. 31, no. 21, pp. 3107-3109, 2006.

[10] I. Kim and K. Y. Lau, "Frequency and timing stability of mode-locked semiconductor lasers," IEEE J. Quantum Electron., vol. 29, no. 4, pp. 1081-1090, Apr. 1993. 